\title{
Sensitivity of VOC Sensors for Air Quality Monitoring within the EURAMET Key-VOC project
}

\author{
L. Spinelle1, M. Gerboles ${ }^{1}$, G. Kok ${ }^{2}$ and T. Sauerwald ${ }^{3}$ \\ ${ }^{1}$ European Commission, Joint Research Centre, Institute for Environment and Sustainability, Ispra, Italy \\ ${ }^{2}$ VSL Dutch Metrology Institute, Delft, the Netherlands \\ ${ }^{3}$ Universitaet des Saarlandes, Saarbruecken, Germany
}

\begin{abstract}
The Air Quality Directive AQD sets the limit value for benzene at $5 \mu \mathrm{g} / \mathrm{m}^{3}$ over a calendar year which corresponds to the upper range of concentration measured in air quality monitoring. Currently, the sensitivity of sensors represents the major challenge for measuring benzene in the ppb range. In 2005, benzene levels in ambient air less than $100 \mathrm{ppb}$ were out of the range of gas sensors. Nowadays, the technological progress resulted in an improvement of sensor sensitivity and a few systems are able to reach the $\mathrm{ppb}$ or more rarely sub ppb level of sensitivity for monitoring benzene. The information available for the sensitivity of gas sensors either commercially available or extracted from research studies are presented.
\end{abstract}

Key words: Benzene, photo ionisation detectors, metal oxide sensors, electrochemical cells, micro GCs, electronic noses

\section{Introduction}

Volatile organic compounds (VOCs) are hazardous compounds that may cause damages to human beings for long time exposure. The principal compounds of interest consists of aromatics such as benzene. In Europe, the monitoring of benzene in ambient air is mandatory according to the European Directive for air quality (AQD [1]). This Directive states that the reference method of measurement shall consist of on-line chromatography [2]. This method is expensive to implement and it requires complex operations. However, the AQD allows using indicative measurements such as microsensors. Low cost sensors represent a big opportunity for developing networks of VOC sensors able to monitor within large areas at limited cost compared to reference measurements.

Ten years ago, benzene levels in ambient air less than $100 \mathrm{ppb}$ were out of the range of gas sensors. Nowadays, the technological allows an improvement in sensitivity and a few systems are able to reach the ppb or sub ppb level for monitoring benzene in ambient air. In fact, the AQD set the limit value (LV) for benzene at $5 \mu \mathrm{g} / \mathrm{m}^{3}$ (about $1.5 \mathrm{ppb}$ ) over a calendar year. Hereafter, the sensitivity and selectivity of gas sensors either commercially available or extracted from research studies are presented.
The type of sensor includes metal oxide, electrochemical cells, photo ionisation detectors, UV spectrometers, micro GCs and electronic noses.

\section{Photo-ionization sensors}

Photo-Ionization Detector (PID) are ion detectors which uses high-energy photons, in the ultraviolet (UV) spectrum to break molecules into positively charged ions. The gas becomes electrically charged and the ions produce an electric current, which becomes the output signal of the detector. The current produced by PIDs is directly proportional to the number of produced ions that depends on the concentration of the gas compound to be measured. PIDs are broad band detectors, lacking selectivity as they ionize all compounds with an Ionisation Potential less than or equal to the lamp beam.

Ion Science Ltd (UK), AlphaSense (UK), and Baseline (USA) are the major Original Equipment Manufacturer (OEM) of PIDs. These small sensors can reach a sensitivity of sub-ppb for benzene with the MiniPiD white, PID-AH and piD-TECH eVx Blue, respectively (see Fig. 1). Even with the lowest available lamp energy (xenon $9.6 \mathrm{eV}$ ) BTX cannot be distinguished between benzene, toluene and xylene as their, Ionisation Potential is lower than the energy of the lamp. A few manufacturers propose portable instrument 


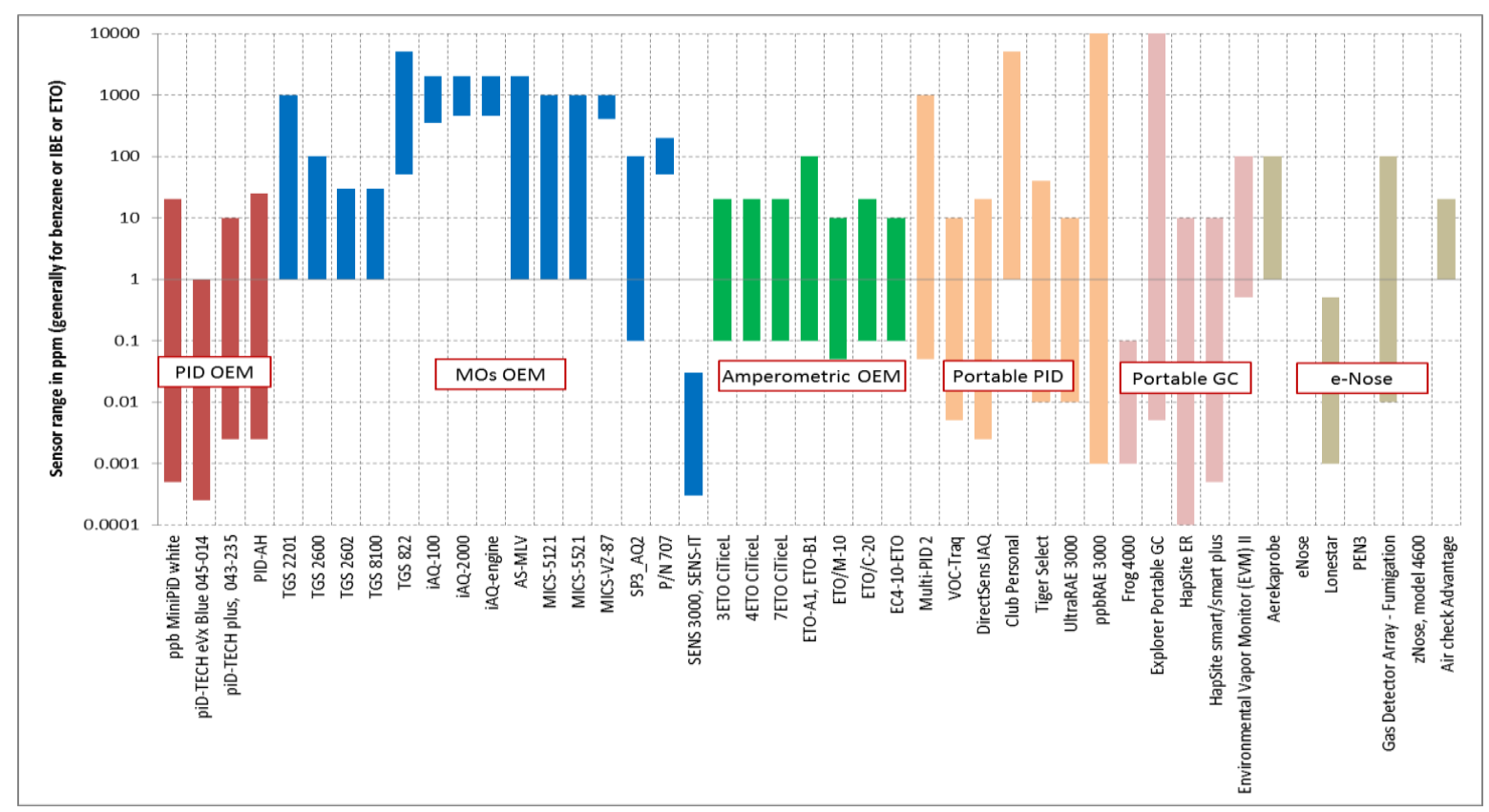

Fig. 1. Commercially available sensors for VOC measurements.

displaying real time measurements (Drager model Multi-PID 2, Baseline-Mocon model VOCTraq, GrayWolf Inc models AdvancedSenseDirectSens, Ion Science Ltd model Club Personal and Tiger Select, and RAE Systems Inc. model UltraRAE 3000 and model ppbRAE 3000). Some of them includes a selective benzene absorbing cartridge (Multi-PID 2, Tiger Select benzene and the UltraRAE 3000). Unfortunately, the sensitivity of the portable instrument is lower than the one of OEM sensors especially for the ones selective to benzene. They reach limits of detection of $50 \mathrm{ppb}$ for the Drager instrument and $10 \mathrm{ppb}$ for the Ion Science and RAE instruments.

\section{Electrochemical cells}

The electrochemical sensors are classified as potentiometric sensors if the output is an electromotive force and as amperometric if the output is a current. Electrochemical reactions are always based on the transfer of a charge from an electrode to another phase (electrolyte), which can be solid, gel-like or liquid. This process comprises a chemical reaction of the electrode as well as charge transport through an electrolyte, which can both be chemically influenced.

The OEM manufacturers propose 3-electrode amperometric sensors for measuring VOC which are generally adjusted to measure ethylene oxide. They also report a number of inorganic and organic interfering compounds. The main sensor models consist of City Technology (UK) 3ETO, 4ETO and 7ETO CiTiceL, Alphasense (UK) ETOA1 and ETO-B1, Membrapor AG (CH) ETO/M-10 and ETO/C-20 and SGX Sensortech (CH) EC4-10-
ETO. All these sensors appear to give a limit of detection that is too high for air quality monitoring with the best value reaching $50 \mathrm{ppb}$. All sensors exhibit similar sensitivity between 1.9 and $2.8 \mu \mathrm{A} / \mathrm{ppm}$ that is too low for measuring $\mathrm{VOC}$ in the ppb or sub ppb range.

\section{Metal oxide sensors}

These sensors consist on a metal oxide (MOx) that changes its electrical properties when exposed to different ambient gases because of change in charge mobility. In commercial sensors the overwhelming property measured in metal oxide sensors is the resistance or conductivity. The tin oxide ( $\mathrm{SnO} 2$ ) is the most used metal oxide because it has a wide reactivity and strong changes of resistance. The model widely accepted is that tin oxide form grains and the boundary of those grains dominate the conductivity. In presence of an oxidizing gas, normally oxygen in ambient air, the molecules of this gas react with the tin oxide trapping electrons of the surface creating a positive charge space that act as a barrier for the conductivity.

A lot of MOx sensors for VOC are commercially available: AMS (S) models iAQ-100, iAQ-2000, iAQ-engine and AS-MLV; Unitec (I) SENS 3000 or SENS-IT; UST (D) GGS-1330T, 3330T, 8330T; SGX Sensortech (CH) MICS-5121, 5521 and VZ-87; Figaro (J) TGS 2201, 2600, 2602, 8100 and 822, FIS (J) SP3_AQ2 and Synkera Technologies (USA) VOC Sensor (P/n 731). As for amperometric sensors, the limit of detection of these sensors is too high for air quality monitoring with the best value reaching $100 \mathrm{ppb}$ excepted for the SENS3000. 
Metal oxide semiconductor (MOS) gas sensors in general respond to a large variety of gases. To achieve selectivity the well-established temperature cycled operation(TCO) can be used [3]. The signal output of TCO can be seen as a virtual sensor array providing multichannel information. Typically discrimination and quantification algorithms like e.g. linear discriminant analysis (LDA) and partial least square regression (PLS) are used for data processing. Commercial sensor using TCO have been shown in lab investigations to detect toxic VOC in the ppb range [4]. Some sensor manufacturer provide information on TCO parameters in their data sheet e.g. Figaro Engineering for the detection of CO (TGS-2442) but in most case the specific use of TCO is implemented by sensor system manufacturers [5]. Sensor systems for VOC detection based on TCO have been reported from several companies e.g. 3S GmbH-Germany and NanoSense,-France. The TCO mode may be also applied to Silicon Carbide Feld Effect Transistors (SiC-FET) that allows to reach sub-ppb sensitivity likely keeping the selectivity through the use of LDA and PLS statistical methods ([6] and [7]).

\section{Gas chromatographic system}

Low power consumption, sample processing, column programming, detection systems, and data handling have been combined to reduce the size and weight of GCs for portable use. The simplest may consist of an ambient temperature injector, column, and detector, while the most complex may have every feature of an advanced laboratory instrument. Portable GCs may be based on semiconductor chip processing or assembled from discrete components. The ion mobility spectrometer (IMS) can be considered as a sub-class of chromatographic separators. The principle of every IMS is a time-of-flight measurement. After a gaseous sample has entered the spectrometer it will be ionized by a radioactive source, the resulting positive and negative charged species will be accelerated over a short distance and the time-of-flight will be determined. The IMS is different from mass spectrometer in that it operates under atmospheric conditions and does not need large and expensive vacuum pumps. Because of this, IMS can be easily miniaturized.

The instruments of this category reach the desired sensitivity and selectivity (Defiant Technologies model Frog 4000, INFICON models Explorer Portable GC, HapSite ER and HapSite smart/smart plus, FemtoScan model EVM II). However, the price of such instruments (between $20 \mathrm{k}$ and $100 \mathrm{k} €$ ) limit their applicability.
A few gas chromatographic micro-system, able to analyse in the sub-ppb range have been developed for BTEX ([8] and [9]) or for other VOCs (e. g. trichloroethylene [10]). For example, the system of ref. [10] consists of a preconcentration unit, a separation unit and a detection unit. The pre-concentration unit is based on a MEMS pre-concentration column that traps aromatic compounds during sampling. When heated, it injects the pollutant into a MEMS packed temperature controlled GC column for separation. The detection unit consist of a MOX sensors array which provides the signals. The air samples and the on-board generated carrier gas flow are provided by 2 mini pumps. Micro systems (lab-on-a-chip) have been developed but are for now limited to the sub-ppm range ([11], [12] and [13]).

\section{Spectrophotometric methods}

In a recent review of research studies for optical and colorimetric-based portable devices for high sensitive and real time BTEX detection[14], two systems were suitable for the low ppb range. The $1^{\text {st }}$ system used a micro-fluidic UV portable spectrometer with a silicate absorbent and subsequent thermal desorption which resulted in a limit of detection of about $10 \mathrm{ppb}$ for hourly values [15]. The system was further improved to reach a limit of detection of about $1 \mathrm{ppb}$ for 30 min averages whilst resulting in a more complicated setup including a pulse pump system and new detection cell [16].

The $2^{\text {nd }}$ prototype was based on the variation of the reflected light intensity when BTEX gases are present in a detection tube holding an optical fiber coated with a polymeric sensitive film [17]. The change in the intensity was proportional to the amount of BTEX present inside the tube. Gaseous BTEX were transmitted to the glass tubing that contains a polydimethysiloxane layer for the adsorption and desorption processes and finally to the detection tube that contained the coated sensitive film optical fiber. The detection limit was found to be around $2.5 \mathrm{ppb}$ for benzene without the need for a pre-concentration step.

\section{E-Nose and sensor arrays}

Another class of measurement devices consists of sensor arrays. Smart pattern recognition software often based on neural networks is used to make the sensor array more specific than the sum of the single sensors. Sensor arrays are often part of devices called "e-noses". Some examples of commercially available sensor arrays are the eNose of Comon-Invent consisting of 4 different semi-conductor sensors, the Aerekaprobe of The eNose Company using a system with 1 to 12 
micro-hotplate temperature modulated metaloxide sensors, and the Airsense GDA-F which is based on a hybrid sensor array combining different detection technologies. The range of measurable gas concentrations of these sensor arrays varies from ppb to upper ppm with limited information about selectivity.

In 2008, a prototype of e-nose built with 7 MOx was presented [18]. After a field neural calibration, the e-nose was shown to be sensitive down to sub-ppb levels, selective and stable over time for the prediction of benzene in urban environment. Estimations of the e-nose using a small number of measurement days were shown to produce limited prediction errors for more than 6th months. Subsequently, the seasonal influences on prediction capabilities at lowconcentrations suggested the need for a further calibration over a total of 13 month monitoring period. Relationship between training length and performances are also investigated.

\section{Conclusion}

Commercial OEMs sensors are generally not enough selective (PID) or sensitive (MOx and amperometric) for measuring benzene and other VOC in the ppb range. Portable PID instruments may be an alternative at level from $10 \mathrm{ppb}$ for the ones selective to benzene (Tiger Select Benzene and UltraRAE3000). Portable GC and IMS that would be both sensitive and selective are simply too expensive for the market of low-cost sensors.

Among research studies, one can find prototypes or commercial systems comprising portable UV spectrometers, and multi MOx sensors or SiC-FET operated in Temperature Operation Cycle that offer both ppb or sub ppb sensitivity with selectivity to specified VOCs. A few advanced prototypes of miniaturized Gas Chromatographs can be found in literature with equivalent or better sensitivity and selectivity.

Finally, multi sensors coupled with artificial neural network algorithms can be also considered a possible candidate sensor system.

\section{References}

[1] 2008/50/EC: Directive of the European Parliament and of the Council of 21 May 2008 on ambient air quality and cleaner air for Europe.

[2] EN 14662:2005, 'Ambient air quality - Ambient air quality - Standard method for measurement of benzene concentrations.

[3] A. Gramm and A. Schütze, "High performance solvent vapor identification with a two sensor array using temperature cycling and pattern classification," Sens. Actuators B Chem., vol. 95, no. 1-3, pp. 58-65, 2003
[4] M. Leidinger et al., Selective detection of hazardous VOCs for indoor air quality applications using a virtual gas sensor array," J. Sens. Sens. Syst., vol. 3, no. 2, pp. 253-263, 2014

[5] D. Kohl et al., Detection of Fires by Gas Sensors, Sens. Update, vol. 9, no. 1, pp. 161-223, May 2001.

[6] D. Puglisi et al., Catalytic metal-gate field effect transistors based on $\mathrm{SiC}$ for indoor air quality control, J. Sens. Sens. Syst., vol. 4, no. 1, pp. 1-8, Jan. 2015.

[7] C. Bur et al., Discrimination and Quantification of Volatile Organic Compounds in the ppb-Range with Gas Sensitive SiC-Field Effect Transistors, Procedia Eng., vol. 87, pp. 604-607, 2014.

[8] S. Zampolli et al., Real-time monitoring of sub-ppb concentrations of aromatic volatiles with a MEMSenabled miniaturized gas-chromatograph, Sens. Actuators B Chem., vol. 141, no. 1, pp. 322-328, 2009.

[9] B. Bae et al., Development of a portable gas analyzer using a micro-Gas Chromatograph/Flame Ionization Detector (micro-GC/FID) for NASA's environmental missions, 2012.

[10] S. K. Kim et al., Microfabricated Gas Chromatograph for On-Site Determination of Trichloroethylene in Indoor Air Arising from Vapor Intrusion. 1. Field Evaluation, Environ. Sci. Technol., vol. 46, no. 11, pp. 6065-6072, 2012.

[11] J.-B. Sanchez et al., A selective gas detection microdevice for monitoring the volatile organic compounds pollution, Sens. Actuators B Chem., vol 119, no. 1, pp. 227-233, 2006.

[12] C. Chen et al., A wireless hybrid chemical sensor for detection of environmental volatile organic compounds, IEEE Sens. J., vol. 13, no. 5, pp. 17481755, 2013.

[13] J. Halliday et al., Lab-on-a-chip GC for environmental research, LC GC Eur., vol. 23, no. 11, 2010.

[14] A. Allouch et al., Portable, miniature, fast and high sensitive real-time analyzers: BTEX detection, Sens. Actuators B Chem., vol. 182, pp. 446-452, 2013.

[15] T. Horiuchi et al., Portable Aromatic VOC Gas Sensor for Onsite Continuous Air Monitoring with 10-ppb Benzene Detection Capability, NTT Tech. Rev., vol. 1, pp. 30-37, 2006.

[16] S. Camou et al., Portable Sensor for Determining Benzene Concentration from Airborne/ liquid Samples with High Accuracy, NTT Tech. Rev., vol. 10 , no. 2, pp. 1-7, 2012 .

[17] L. I. B. Silva et al., Polymeric nanofilm-coated optical fibre sensor for speciation of aromatic compounds, Int. J. Environ. Anal. Chem., vol. 89, no. 3, pp. 183-197, 2009.

[18] S. De Vito et al., On field calibration of an electronic nose for benzene estimation in an urban pollution monitoring scenario, Sens. Actuators B Chem., vol. 129, no. 2, pp. 750-757, 2008. 